
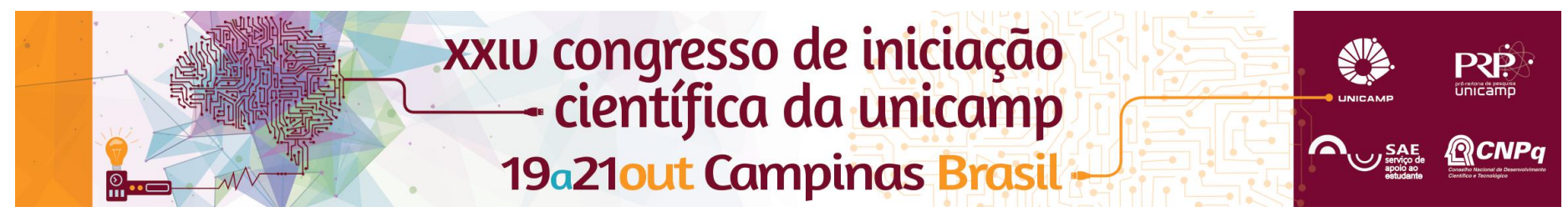

\title{
Hydrophobic Glasses Surfaces Modified With Iron Oxide
}

\author{
Gabriela Thais Augusto da Silva*, Celso Aparecido Bertran.
}

\begin{abstract}
The present research is related to the Surface Science, mainly concerned with hydrophobic and superhydrophobic surfaces. The purpose of this work is basically to obtain a modified glass surface with high contact angle what is commonly found in materials with these properties. The first scientific demonstration of an artificial superhydrophobic surface was in 1990 decade $^{1}$, when scientists could reproduce a characteristic found in some plants: their leaves showed specific roughness and hydrophobicity, so that, the droplets acquired a spherical shape when touched the leaves surfaces. Nowadays, this characteristic is called "The Lottus Effect"2. Intending to increase the glass hydrophobicity by increasing the contact angle between glass and water, iron oxide nanoparticles were incrusted on glass to obtain a texturized surface and reactive chemical sites that allowed also chemical modifications.
\end{abstract}

\section{Key words:}

Hydrophobic and Superhydrophobic surfaces, Iron Oxide modified surfaces, contact angle

\section{Introduction}

Since a sphere has the smallest ratio volume/surface area, a volume of a liquid that can freely adjust its shape tends to acquire a spherical shape to minimize the surface free energy. However, the gravity and the interaction between the liquid and the solid are some of the aspects that also affect the contours of a liquid volume ${ }^{3}$.

As higher the wettability of a surface, lower is its hydrophobicity. The wettability is mainly determined by factors as the surface energy and surface roughness, since that the contact angle increases as the surface energy decreases ${ }^{4}$ and also when roughness at nanoscale or micro-scale is present. ${ }^{5}$

These principles guided the objectives of this project, where the fixation of iron oxide particles on glasses surfaces can result in hydrophobicity, both by the roughness that these particles imply or by the possibility to chemically bound hydrophobic substances on the particles.

\section{Results and Discussion}

Initially, it was necessary to synthesize particles of iron oxide in nanoscale or micro-scale to deposit them on the glass surface, what was reached by adding, under constant heating, concentrated hydrogen peroxide in a ferric chloride solution which had its $\mathrm{pH}$ adjusted slowly by ammonia gas.

The glass line used was previously treated by dipping it in melted sodium hydroxide at $400{ }^{\circ} \mathrm{C}$ for 15 minutes to produce Hydroxyl groups on the surface. After lamina wash, it was put in the iron oxide suspension at its boiling for about 15 minutes. Next, this glass with iron oxide deposit was heated for 120 minutes at $500{ }^{\circ} \mathrm{C}$ to provide the adhesion of iron particles on glass. After that time, the sample was cleaned by water and common detergent. The particles adhesion on the surfaces was determined by scanning electron microscopy.
To modify chemically the surface some thiol and fluorinated hydrophobic compounds were tested.

Although, the iron oxide particles were strongly adhered on the glass surface, the chemical modification by thiol and fluorinated compounds failed, and even so, the surface still quite hydrophilic.

\section{Conclusions}

The coverage of iron oxide was not homogeneous on the surface although the particles were strongly adhered. However, the particles alone the related roughness are not enough to increase the contact angle and the hydrophobicity of a glass surface.

The compounds tested were chosen due to the possibility of the reaction of the thiol and fluoride groups with iron oxide, although they had not reduced appreciably the surface-liquid interaction as thought.

It is important to find a chemical compound that reacts with the iron oxide besides providing a contact angle increase, so that the substance needs to have affinity with interfaces.

\footnotetext{
${ }^{1}$ MA, M.; HILL, R.M. Superhydrophobic surfaces. Current Opinion in Colloid\&Interface Science, v.11, p.193-202, 2006.

${ }^{2}$ SHIRTCLIFF, N. J.; MCHALE, G.; ATHERTON, S.; NEWTON, M. I. An introduction to superhydrophobicity. Advances in Colloid and Interface Science, v.161, p.124-138, 2010.

${ }^{3}$ SHIRTCLIFF, N. J.; MCHALE, G.; ATHERTON, S.; NEWTON, M. I. An introduction to superhydrophobicity. Advances in Colloid and Interface Science, v.161, p.124-138, 2010.

${ }^{4}$ MIWA, M. NAKAJIMA, A.; FUJISHIMA, A.; HASHIMOTO, K.;

WATANABE, T. Effects os the surface Roughness on Sliding Angles of Water Droplets on Superhydrophobic Surfaces. Lagmuir, v.16, p.5754-5760, 2000.

${ }^{5}$ MA, M.; HILL, R.M. Superhydrophobic surfaces. Current Opinion in Colloid\&Interface Science, v.11, p.193-202, 2006.
} 